\title{
W1B-(2)-3
}

\section{Improved temperature characteristics of semiconductor lasers due to carrier redistribution among nonidentical multiple quantum wells}

\author{
Chao-Hsin Wu, Di-Ku Yu, Ching-Fuh Lin* \\ Institute of Electro-Optical Engineering, National Taiwan University, Taipei, Taiwan, \\ Republic of China \\ "also with Institute of Electronic Engineering and Department of Electronic Engineering \\ Phone: 886-2-23635251 ext 339, Fax: 886-2-23677467, Email: cflin@cc.ee.ntu.edu.tw
}

\begin{abstract}
Carriers among nonidentical multiple quantum wells (MQWs) will redistribute as temperature varies. This is due to strongly temperature-dependent Fermi-Dirac distribution, which favors carriers in high energy states in high temperature. As a result, the temperature characteristic of semiconductor can be improved.
\end{abstract}

\section{INTRODUCTION}

For laser diodes with nonidentical MQWs, the temperature-induced carrier redistribution could be observed by different experimental results. It causes the lasing wavelength much less dependent on temperature, compared to the bandgap shrinkage. We also find that there are two lasing wavelength peaks in the spectrum of this laser diode at room temperature. The relative amplitude of these two lasing peaks will change as ambient temperature increases. Meanwhile, the relationship of threshold current and temperature at two different lasing wavelengths shows that more carriers move to short wavelength $\mathrm{QW}$ at high temperature.

\section{THEORETICAL BACKGROUND}

Assume that the quantum-well (QW) structure has only two different $\mathrm{QWs}$. QW $\mathrm{A}$ has one quantized energy at $\mathrm{E} 1$ and $\mathrm{QW} B$ has one quantized energy at E2, and $\mathrm{E} 1<\mathrm{E} 2$. For a certain injection level of carriers, the Fermi level $\left(E_{F}\right)$ is assumed to be slightly above energy $E_{2}$. As temperature increases, carriers in $Q W A$ and in QW B change the different numbers respectively because of the Fermi-Dirac distribution varies with temperature. For a certain injection current, the total amount of carriers in the QWs is approximately constant. Thus carriers flow from QW A to QW B as temperature increases and vise versa as temperature decreases.

\section{EXPERIMENT}

The QW structure for the experiment has two In0.53Ga0.47As QWs near the p-cladding layer and three In 0.67Ga0.33As0.72P0.28 QWs near the n-cladding layer. The $\mathrm{QWs}$ are separated by $\mathrm{In} 0.86 \mathrm{Ga} 0.14 \mathrm{As} 0.3 \mathrm{P} 0.7$ barriers. At room temperature, the In0.53Ga0.47As QWs (QW A) and In0.67Ga0.33As0.72P0.28 QWs (QW B) have their first quantized transition energies of $0.8 \mathrm{eV}$ and $0.954 \mathrm{eV}$, respectively. Because the bandgap shrinks with temperature, those transition energies decrease with temperature. When we put theoretical values and experimental values together, we find the measured wavelength of the above laser diode shows much less temperature dependence. For temperature varying from $33 \mathrm{~K}$ to $260 \mathrm{~K}$, its corresponding energy changes less than $5 \mathrm{meV}$, while the bandgap energy changes more than $50 \mathrm{meV}$.

It is interesting that when we increase the ambient temperature, the spectrum of the device has double lasing peaks. One is at $1365 \mathrm{~nm}$ and the other at $1418 \mathrm{~nm}$. The short wavelength peak emerges and the spacing between these two lasing peaks is quite large. It is significant because it means more carriers move to the 1.3um quantum well at high temperature. If we use a grating in an external cavity to control the lasing wavelength, we see that there is a higher characteristic temperature $T_{0}$ at short wavelength. The measurement of threshold currents for these two lasing wavelengths reveals the relative modal gain of two different quantum wells. When the ambient temperature is lower than $24^{\circ} \mathrm{c}$, light at $1418 \mathrm{~nm}$ has lower threshold current. If the temperature is increased up to $24^{\circ} \mathrm{c}$, light at $1368 \mathrm{~nm}$ has lower threshold current. We also see that the light of $1368 \mathrm{~nm}$ has "minus characteristic temperature" from $21^{\circ} \mathrm{c}$ to $24^{\circ} \mathrm{c}$. It could be the result of the carriers transferring between two quantum wells and the competition between two lasing peaks.

\section{CONCLUSION}

Using nonidentical MQWs structure, temperature sensitivity of long-wavelength semiconductor lasers can be efficiently reduced. Carrier redistribute when temperature increases due to temperature-dependent Fermi-Dirac distribution. In nonidentical MQWs, carriers favor short wavelength $\mathrm{QWs}$ as ambient temperature increases. The temperature-induced carrier redistribution among nonidentical MQWs has been observed to contribute larger characteristic temperature and less temperature dependence compared to conventional InGaAsP/InP semiconductor lasers. With the less temperature-sensitive QW located in the proper location, significant improvement on temperature characteristics of semiconductor lasers is possible. 